\title{
An Adaptive Localized Scheme for Energy-Efficient Broadcasting in Ad Hoc Networks with Directional Antennas ${ }^{\star}$
}

\author{
Julien Cartigny ${ }^{1}$, David Simplot-Ryl ${ }^{1}$, and Ivan Stojmenović ${ }^{2}$ \\ 1 IRCICA/LIFL, University of Lille 1, INRIA Futurs, France. \\ \{cartigny, simplot\}@lifl.fr. \\ 2 SITE at the University of Ottawa, Canada. ivan@site.uottawa.ca.
}

\begin{abstract}
Several solutions for energy-efficient broadcasting, mostly centralized, have been proposed with directional antennas. However, such globalized protocols are not suitable for ad hoc networks, because each node needs a full knowledge of the topology. Recently, a localized algorithm, called DRBOP, using one-to-one communication model has been proposed. It uses RNG graphs, which can be locally computed by each node. However, for energy consumption reasons, it can be useful to reach more than one neighbor at a time. In this paper, we propose an efficient protocol which uses both one-to-one and one-to-many communication models. First, we present a variant of DRBOP efficient for sparse networks, based on LMST graph which is a local adaptation of minimal spanning tree. Then, a one-to-many protocol efficient for dense networks is proposed. From these two algorithms we derive an adaptive protocol which is shown to be efficient for both sparse and dense networks.
\end{abstract}

\section{Introduction}

In mobile wireless ad hoc networks, each node participates in networking tasks by relaying messages, in order to provide a full coverage of the network. This implies a high energy consumption of the radio interface and thus limits the lifespan of the battery unit. The problem of energy consumption is very significant in this type of network, because nodes are energy limited. Many solutions have been proposed to decrease the energy consumption. An idea is to control the emitted transmission power by decreasing the range of the radio beam, and thus reducing the energy consumption [1. Special devices, like directional antennas, offer better energy savings by reducing the angle of the beam, to only cover part of the neighborhood. However, theses adjustments have to be managed while maintaining the connectivity of the network.

There exist several communication models. In one-to-all model, nodes use omnidirectional antennas to cover all their neighbors. In one-to-many model, a

\footnotetext{
* This work was partially supported by a grant from Gemplus Research Labs., an ACI Jeunes Chercheurs "Objets Mobiles Communicants" (1049CDR1) from the Ministry of Education and Scientific Research, France, the CPER Nord-Pas-de-Calais TACT LOMC C21 and NSERC.
} 
node can target a subset of its neighborhood with one transmission by choosing direction and width of the beam, using directional antennas. The one-to-one model is a particular case of the previous one, where nodes use a constant narrow beam to transmit toward a particular neighboring node. Since the communication area is a narrow beam with a small angle, the directional antennas provide more energy saving and interference reduction.

Several solutions have been proposed [2], 3] for energy-efficient broadcasting with directional antennas. However, they are globalized, meaning either a centralized entity has to gather knowledge of the full topology and diffuse information to organize the network, or each node has to know the total topology of the network to locally compute the broadcast spanning tree. This approach is not efficient in ad hoc networks, because of a high communication overhead. We are interested in localized protocols, which require only information about the neighborhood. Furthermore, we are looking to use directional antennas for the one-to-one and one-to-many models.

The problem of finding a broadcast spanning tree with minimal power, and the problem of broadcasting a message with a minimal number of retransmissions are well-known to be both NP-complete [4. Consequently, several heuristics have been proposed. For instance, MST (Minimum Spanning Tree) [1] is a globalized algorithm which builds the spanning tree by choosing shortest links between nodes. The BIP (Broadcast Incremental Power) algorithm [1] (and the directional version DBIP [3]), proposed by Wieselthier et al. is a globalized greedy algorithm inspired by Prim's algorithm, known to be the most efficient existing broadcast protocol. Some localized solutions also exist. For instance, RBOP (RNG Broadcast Oriented Protocol) [5] (and the directional version DRBOP [6]) is a localized energy-efficient broadcast protocol, which only requires local information to construct a RNG subgraph (Relative Neighborhood Graph) [7] while keeping the network connected. Another recent solution is BLMST, Broadcast with Local Minimum Spanning Tree) 8] (and a similar independently proposed solution in [9]) is a localized energy-efficient broadcast algorithm for omnidirectional antennas. It is based on LMST (Local Minimum Spanning Tree) 10, a MST algorithm applied on the local neighborhood.

In this paper, we propose DLBOP (Directed LMST Broadcast Oriented Protocol), an algorithm based on LMST and using directional antennas. This protocol is a straightforward variation of DRBOP [6]. A second algorithm, called OM-DLBOP (One-to-Many Directed LMST Broadcast Oriented Protocol), is used instead of DLBOP when energy cost of one-to-one transmissions is too high (i.e. when network is dense). These two algorithms are combined in a hybrid protocol, called A-DLBOP (Adaptive Directed LMST Broadcast Oriented Protocol), which adaptively decides which communication model to use among one-to-one and one-to-many models. This protocol is energy-efficient and applied on general energy model (proposed in [6]) to optimize the energy consumption. Each node requires only the knowledge of neighbor positions. This information can be measured by using signal strength or time delay combined with direction evaluation by smart antennas. The position of each node can be also extracted with positioning system (like GPS). 
The paper is organized as follows. Some preliminaries are given in Section 2 We describe the existing works on energy-saving broadcast in Section 3. Protocols and their consumption estimations are given in Section 4 The experimental results and the comparison with theoretical bounds and other protocols are proposed in Section 5. Finally, Section 6] concludes the paper.

\section{Preliminaries}

In the unit graph model, two nodes can communicate if and only if their distance is $\leq R$, where $R$ is the transmission radius, equal for all nodes. We denote by $N(u)$ the set of neighbors of $u$.

In one-to-all communication model (omnidirectional antenna), each node in the network can only change its transmission power. In one-to-one and one-tomany models, we will assume that all the nodes have directional antennas. They can hear messages from every neighbor and send messages to every neighbor in unicast communications, by aim the beam to the addressee.

We need to evaluate the energy consumption for each node. We use the following formula proposed in [6]. The cost of a transmission of range $r$ with angle $\theta$ is calculated by:

$$
e(\theta, r)= \begin{cases}\frac{\theta}{2 \pi}\left(r^{\alpha}+C_{1}\right)+C_{2} & \text { if } r \neq 0, \\ 0 & \text { otherwise }\end{cases}
$$

This model generalizes several energy consumption models proposed in the literature. The parameter $\alpha$ gives the power loss. The constant $C_{1}$ associates a energy cost for aiming the angle beam. The constant $C_{2}$ is a constant overhead for each sending, representing the minimum needed energy for signal processing and MAC control mechanism, and the power needed for neighboring nodes to receive the message. Although this power is spent by neighbors, we simplified the model by charging the node itself, assuming that each node eventually still cares about receiving one full copy of the packet, and can decide to switch off the receiver at the beginning of subsequent reception of the same message. For constants $\alpha=2$ and $C_{1}=C_{2}=0$, the model is a generalization of the one commonly used. We considered in [6] a directional version of a specific model proposed by Rodoplu and Meng in [11. This model uses the following constants $\alpha=4, C_{1}=8.10^{7}$ and $C_{2}=2.10^{7}$, thus charging high cost for each directional transmission. In one-to-one model, the beam angle is a constant value while it varies between a minimal angle $\beta$ and $2 \pi$ in one-to-many model.

\section{Literature Review}

Wieselthier et al. proposed two extensions of the BIP protocol [1] with directional antennas. The protocol BIP is an omnidirectional protocol which constructs a spanning tree with respect to the energy consumption. For each step, the algorithm decides if the best solution is to create a new transmission beam or to increase the range of an existing transmission. The directional version 3 of BIP 


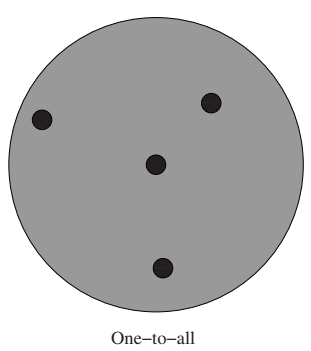

One-to-all

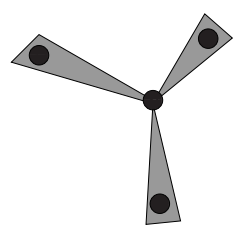

One-to-one

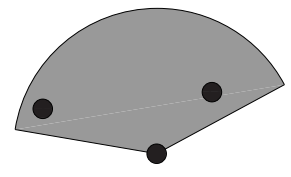

One-to-many

Fig. 1. One-to-all, one-to-one and one-to-many communication models.

proposes two protocols. The first protocol is called RB-BIP (Reduced Beam BIP) and uses one-to-one communication model (with minimal angle) to join neighbors in the BIP tree. Because of the tree construction, the BIP spanning tree is exactly the same as the tree built by the MST algorithm. The second protocol is D-BIP (Directional BIP). Each node can send only one message by broadcast, the protocol has to decide, at each step, if it is better to extend the beam and/or the range of a node, or to add a new communication beam. This decision is made with respect to the energy consumption. Hence, if the constants $C_{1}$ and $C_{2}$ are not null, the natural tendencies of D-BIP are to favor transmissions with large radii and beam angles, to avoid retransmissions by every node.

Cartigny et al. have proposed RBOP (RNG Broadcast Oriented Protocol) [5], a localized broadcast protocol for reducing energy consumption with omnidirectional antennas. Each node constructs an RNG (Relative Neighborhood Graph) 7 subgraph from its neighboring graph. Let $G=(V, E)$ be a graph. The RNG subgraph, denoted by $R N G(G)=\left(V, E_{r n g}\right)$, is defined by:

$$
E_{r n g}(G)=\{(u, v) \in E \mid \nexists w \in V \quad d(u, w)<d(u, v) \wedge d(v, w)<d(u, v)\} .
$$

RNG has several advantages: each node needs to know only its neighbors and the distance between them. Furthermore, the required information can be gathered in a localized manner. The RNG transformation removes some edges from the set $E$. In obtained graph, the average degree of nodes is approximately 2.6 , and connected neighbors are the closest neighbors of the node. RNG preserves connectivity. The protocol RBOP consists of a Neighbor Elimination Scheme (NES) [12, 13] limited to RNG neighbors where a transmitting node adjusts its communication range to reach all non-covered RNG neighbors. In a NES proto$\mathrm{col}$, each node eliminates from the list of neighboring nodes for retransmission those nodes that are supposed to receive the same packet received by given node one or more times in previous retransmissions.

A directional version of this protocol, called DRBOP (Directional RNG Broadcast Oriented Protocol), has been proposed in [6]. This algorithm proposes that each node sends a separate unicast message to each of its non-covered RNG neighbors. The protocol is efficient and gives results reasonably close to the cen- 

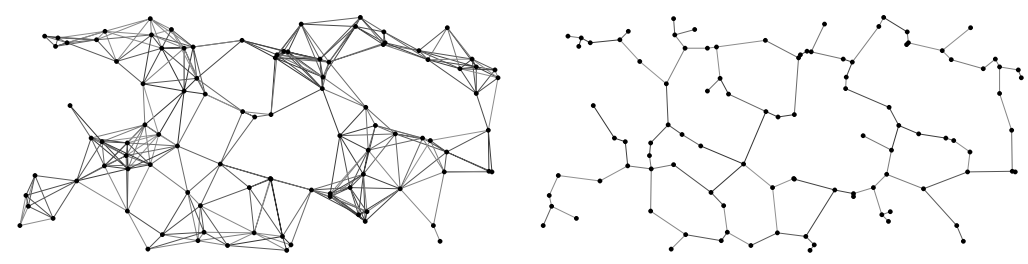

Fig. 2. Sample of network with its LMST subgraph (average degree 10).

tralized MST protocol because DBIP protocol has an average neighbor degree of 1.99 (which is average degree of a minimal spanning tree), and DRBOP has average degree about 2.6, as found by experiments.

Li et al. 10] have proposed LMST (Local Minimum Spanning Tree) which offers a better graph reduction than RNG, with a degree of approximately 2.04 . The LMST method is simple: each node applies MST algorithm on its local topology (the list of neighbors and links between them), and keeps only links that are present in LMST of both endpoints. The LMST algorithm is localized and offers a lower subgraph degree than RNG (in fact, LMST is a subgraph of RNG). Both LMST and RNG require 2-hops informations to be computed. Experimentally, the LMST degree is approximately 2.04, which is closer to the BIP degree (1.99) than RNG (2.6). The authors proposed an omnidirectional protocol for topology control using LMST and proved the correctness of the algorithm for preserving the connectivity. They also proposed BLMST, an energy-saving broadcast protocol using LMST in 8] (a similar protocol is also proposed in 9]). An example of a graph and its LMST subgraph are presented in Figure 2

\section{One-to-One and One-to-Many Protocols}

First, we are going to present DLBOP, a variant of DRBOP based on LMST graph which is a local adaptation of minimal spanning tree. This protocol is shown to be efficient for sparse networks. Then, we present OM-DLBOP, a protocol using one-to-many communications. It is close to the omnidirectional case and shown to be efficient for dense networks. Finally, we present A-DLBOP, an efficient adaptive protocol which uses both one-to-one and one-to-many communication models which is shown to be efficient for both sparse and dense networks.

\subsection{Directed LMST Broadcast Oriented Protocol (DLBOP)}

The directed LMST broadcast oriented protocol (DLBOP) is a variant of DRBOP where the RNG graph is replaced by the LMST set. DLBOP uses one-toone communication model. Hence, each node $u$ sends to its LMST neighbors $v$ an unicast message, with a beam of angle $\beta$ and range $d(u, v)$. This scheme is more efficient with LMST than RNG, because the average degree of LMST (2.04) is lower than the one of RNG (2.6). Thus, each node has to send approximately 
one message (RNG needs one message and half on average), since each node only covers LMST neighbors which have not received the broadcast message. In order to achieve NES with directional antenna, a node $u$, which decides to retransmit the broadcasted message to a given subset $A$ of its neighborhood with one or several beams, includes its position and the beams characteristics which cover nodes from $A$. Hence a node $v$ which receives the message from $u$ can remove nodes of $A$ from its NES list.

The energy consumption of the DLBOP protocol with directional antennas can be derived by summing the power expenditures of one-to-one messages from each node. A node sends a unicast message to each of its LMST neighbors with a beam of minimal angle $\beta$. Since the degree of each node is approximately 2 on the LMST subgraph (including the local forwarder of the broadcast message) we can expect that each node will broadcast in average one unicast message. Let $d_{l m s t}$ denotes the average distance between LMST neighbors. The energy consumption of the DLBOP protocol is approximately:

$$
E_{D L B O P}=n \times e\left(\beta, d_{l m s t}\right) .
$$

Note that this formula does not follow from our general model, and the approximation assumes that each node has degree two in LMST, which is not the exact distribution. However, we made simplification to have simple theoretical estimates, verified by experiments. To evaluate the energy consumption with this model, it is necessary to know the average distance between LMST neighbors, with respect to the area $S$ and the number of nodes $n$. We propose to approximate LMST distance by the length of the edge of regular hexagonal mesh of $n$ nodes covering the area $S$. In a regular hexagonal mesh, we observe that a node is located at the intersection of three hexagons, and thus two nodes are needed for each hexagon. The size of a hexagon side is then:

$$
d_{l m s t} \simeq r_{h e x}=\sqrt{\frac{4 S}{3 \sqrt{3} n}} .
$$

This assumption can be verified experimentally. The Figure 3 presents the graph of the average distance in LMST from the theoretical and experimental point of view $(S=2000 \times 2000 \mathrm{~m}$ and $R=250 \mathrm{~m})$. Although if the experimental data are lower (because of the border effect), they have the same behavior

Finally, the energy consumption formula (1) can be rewritten, using the formula (2), as:

$$
E_{D L B O P}=n\left(\frac{\beta}{2 \pi}\left(\left(\frac{4 S}{3 \sqrt{3} n}\right)^{\frac{\alpha}{2}}+C_{1}\right)+C_{2}\right)
$$

\subsection{One-to-Many Directed LMST Broadcast Oriented Protocol (OM-DLBOP)}

The DLBOP algorithm has high energy consumption if $C_{1}>0$ and $C_{2}>0$. Hence, in this case it can be beneficial to reach several neighbors with the same 


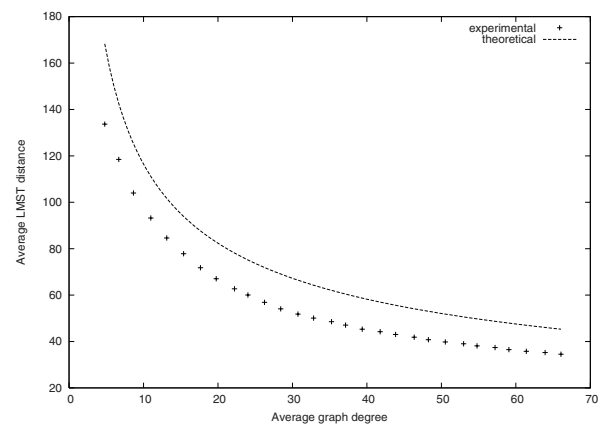

Fig. 3. Average distance between lmst neighbors, from the theoretical and experimental point of view.

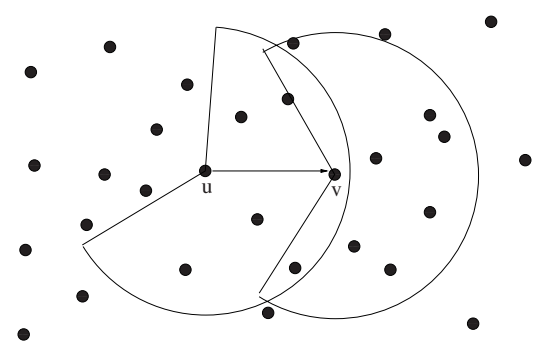

Fig. 4. Beam coverage with OM-DLBOP broadcast.

beam. The one-to-many variant of DLBOP, denoted by OM-DLBOP, consists of sending a single variable angle beam instead of several narrow beams. A node which decides to retransmit the message - because of LMST neighbor elimination scheme reason - uses a single beam with an appropriate angle which allows reaching non-covered LMST neighbors.

To increase energy savings, it can be useful to extend the range in order to avoid excessive retransmissions that can be expensive if constants $C_{1}$ or $C_{2}$ are not null. In fact, it is not always efficient to minimize transmission range because the reduction of transmission range implies a greater number of transmissions in the entire network. This property is also observed in one-to-all communication model 14. For instance, let us consider the broadcasting by uniform beams with $\gamma$ angle $(\gamma \in[\beta, 2 \pi]$ where $\beta$ is the minimal angle) and range $r$. For a given angle $\gamma$, we look for an optimal radius $R_{o p t}(\gamma)$ which minimizes the total energy consumption.

To cover the circular area around itself, a node needs to send $2 \pi / \gamma$ beams with the cost $e(\gamma, r)$ associated with each beam. Suppose that the full area $S$ is ideally covered with such beams (of course, this is impossible but nevertheless leads to useful conclusions). The total energy consumption, denoted by $E_{\text {area }}(r)$ 
is then:

$$
E_{\text {area }}(r)=\frac{S}{\pi r^{2}} \frac{2 \pi}{\gamma} e(\gamma, r)=\frac{S}{\pi}\left(r^{\alpha-2}+C_{1} r^{-2}+\frac{2 \pi C_{2} r^{-2}}{\gamma}\right) .
$$

The behavior of the function $E_{\text {area }}(r)$ depends on $\alpha, C_{1}$ and $C_{2}$ and is summarized in Table 1]. The formula for the optimal radius is obtained by standard calculus method of finding the root of the first derivative over $r$. Interestingly, the optimal radius does not depend on the node density or average node degrees. However, it is valid only for reasonably dense networks since otherwise sparse networks may have large portions of empty zones that do not need coverage.

Table 1. Behavior of total energy consumption.

\begin{tabular}{c|c|c} 
& $C_{1}=C_{2}=0$ & $C_{1} \neq 0 \vee C_{2} \neq 0$ \\
\hline$\alpha=2$ & constant & monotone \\
& decreasing \\
& No $R_{\text {opt }}(\gamma)$ & $R_{o p t}(\gamma)=R$ \\
\hline & monotone & minimal at \\
$\alpha>2$ & increasing & $r(\gamma)=\sqrt[\alpha]{\frac{2 C_{1}+\frac{4 \pi C_{2}}{\gamma}}{\alpha-2}}$ \\
& $R_{\text {opt }}(\gamma)=0$ & $R_{\text {opt }}(\gamma)=\min (r(\gamma), R)$
\end{tabular}

Now, we give the complete OM-DLBOP algorithm. We choose to send beams with angle $4 \pi / 3$. This angle minimizes the overlap communication zone and provides a good coverage of the neighborhood as illustrated Figure 4 (in case of uniform transmission range). Note that the beam can be wider than $4 \pi / 3$ as explained below. The angle of $4 \pi / 3$ represents the ideal case and is used to determine the transmission range. The beam angle chosen by $v$ is set symmetrically with respect to the line $u v$ as shown in the same figure because, since the angle between any two LMST neighbors is at least $\pi / 6$, the mentioned beam contains the remaining LMST neighbors of $v$. LMST neighbors that already received the same message can be determined from the position of sender, the positions of the neighbors and the transmission range and the beam direction of sender. After applying NES restricted to LMST neighbors, a node $u$ which decides to retransmit computes its transmitting angle and range as follows:

- Let $A$ be the set of uncovered neighbors and $B \subseteq A$ the set of uncovered LMST neighbors.

- The node $u$ computes the set of nodes closer than $R_{o p t}(4 \pi / 3)$ :

$$
A^{\prime}=\left\{v \in A \mid d(u, v) \leq R_{o p t}(4 \pi / 3)\right\} .
$$

The "goal" of $u$ is to reach nodes of $C=A^{\prime} \cup B$, i.e. nodes closer than $R_{\text {opt }}(4 \pi / 3)$, for optimization reason, and covering LMST neighbors, for coverage reason. If $C_{1}=C_{2}=0$, the optimal radius cannot be evaluated or is null. In this case, we always consider $R_{\text {opt }}(4 \pi / 3)=0$. This implies that $A^{\prime}=\emptyset$ and the sender must cover only its non-covered LMST neighbors. 
- The node calculates the angle $\theta$ needed to cover $C$ and the distance $d$ to the furthest node of $C$. If $\theta<\beta$ then set $\theta=\beta$. If $C$ is empty, the retransmission is canceled.

- If $d>R_{\text {opt }}(\theta)$ then send $\theta$-beam with $d$ range. Otherwise, the node sets the range of $\theta$-beam (without modifying the orientation) in order to reach all nodes of $A$ closer than $R_{o p t}(\theta)$ (thus the selected radius is generally somewhat lower than $\left.R_{o p t}(\theta)\right)$.

An evaluation of energy consumption of OM-DLBOP can be obtained if we consider an ideal $4 \pi / 3$ beam tessellation. Let us consider an area $S$ with $N$ relaying nodes. Consider an approximation where relaying nodes are placed according to a honeycomb mesh. Suppose that the area $S$ is divided into hexagons with side $R_{\text {opt }}=R_{\text {opt }}(4 \pi / 3)$. Then $N=2 S / A_{\text {hex }}$ where $A_{\text {hex }}=3 R_{\text {opt }}^{2} \sqrt{3} / 2$. Therefore the energy consumption is then

$$
\begin{aligned}
E_{O M-D L B O P} & =N \times e\left(4 \pi / 3, R_{o p t}\right) \\
& =\frac{8 S}{9 \sqrt{3}}\left(R_{o p t}\right)^{\alpha-2}+\frac{4 S}{3 \sqrt{3}}\left(\frac{2}{3} C_{1}+C_{2}\right) R_{o p t}-2 .
\end{aligned}
$$

A comparison between $E_{O M-D L B O P}$ and $E_{\text {area }}(r)$ gives the same behavior, as seen in Table 1:

- If $\alpha=2$ and $C_{1}=C_{2}=0$, the optimal radius does not matter, as the energy consumption of OM-DLBOP is $E_{O M-D L B O P}=\frac{8 S}{9 \sqrt{3}}$.

- With the case $\alpha=2, C_{1} \neq 0$ and $C_{2} \neq 0$, it is shown in the Table 1 that the best solution is to maximize $R_{o p t}$. This is confirmed by the value of $E_{O M-D L B O P}$, equals to $E_{O M-D L B O P}=\frac{8 S}{9 \sqrt{3}}+\frac{4 S}{3 \sqrt{3}}\left(\frac{2}{3} C_{1}+C_{2}\right) R_{\text {opt }}{ }^{-2}$. And so, the energy consumption with $R_{o p t}=R$ is $E_{O M-D L B O P}=\frac{8 S}{9 \sqrt{3}}+$ $\frac{4 S}{3 \sqrt{3}}\left(\frac{2}{3} C_{1}+C_{2}\right) R^{-2}$.

- If $\alpha>2$ and $C_{1}=C_{2}=0$, as presented by the Table 1, it is better to minimize the range. This is confirmed by the rewriting of $E_{O M-D L B O P}=$ $\frac{8 S}{9 \sqrt{3}} R_{o p t}{ }^{\alpha-2}$. The best solution is to use the minimal range in the graph, so $R_{\text {opt }}=d_{l m s t}$. Hence, the energy consumption can be write as

$E_{O M-D L B O P}=\frac{8 S}{9 \sqrt{3}} d_{l m s t}^{\alpha-2}=\frac{2 n}{3}\left(\frac{4 S}{3 \sqrt{3} n}\right)^{\frac{\alpha}{2}}$.

- For the last case $\alpha>2, C_{1} \neq 0$ and $C_{2} \neq 0$, the original equation (4) has no simplification.

\subsection{Looking for a Threshold}

It seems that the two approaches (one-to-one and one-to-many) are valid. The protocol DLBOP offers a subgraph with a minimal degree for each node, and OM-DLBOP covers large group of nodes to reduce the cost associated with each sending. We are now developing theoretically what will be the energy consumption, and showing which protocol is better in respect of the selected energy consumption model. 
For the four energy models, we investigate now the cases when $E_{O M-D L B O P}$ is performing better than $E_{D L B O P}$. The inequality $E_{O M-D L B O P}<E_{D L B O P}$ is resolved by using the formula (4) and (3) with the values of $\alpha, C_{1}$ and $C_{2}$ of the energy models.

- For $\alpha=2, C_{1}=0$ and $C_{2}=0, E_{O M-D L B O P}<E_{D L B O P}$ if and only if $4 \pi / 3<\beta$.

- With $\alpha=2$ and $C_{1} \neq 0$ or $C_{2} \neq 0$, the inequality $E_{O M-D L B O P}<E_{D L B O P}$ is true if and only if:

$$
d>\frac{4 \pi\left(\left(\frac{2}{3}-\frac{\beta}{2 \pi}\right) R^{2}+\frac{2}{3} C_{1}+C_{2}\right)}{3 \sqrt{3}\left(\frac{\beta}{2 \pi} C_{1}+C_{2}\right)},
$$

where $d$ is the density in number of nodes per communication area. For a density higher than this threshold, the best solution is to use the OMDLBOP algorithm. Otherwise, it is better to use DLBOP.

- For $\alpha>2, C_{1}=0$ and $C_{2}=0, E_{O M-D L B O P}<E_{D L B O P}$ if and only if $4 \pi / 3<\beta$.

- With $\alpha>2$ and $C_{1} \neq 0$ or $C_{2} \neq 0$, because $E_{O M-D L B O P}$ is a constant (it does not depend of $n$ and $\beta$ is fixed to $4 \pi / 3)$, the inequality $E_{O M-D L B O P}<$ $E_{D L B O P}$ can be reduced as:

$$
n^{\left(1-\frac{\alpha}{2}\right)} \frac{\beta}{2 \pi}\left(\frac{4 S}{3 \sqrt{3}}\right)^{\left(\frac{\alpha}{2}\right)}+n\left(\frac{\beta}{2 \pi} C_{1}+C_{2}\right)>E_{O M-D L B O P} .
$$

The value $E_{O M-D L B O P}$ is a constant, whatever is the number of nodes. This is a polynomial, whose solutions will be described in the next section.

In DLBOP and OM-DLBOP, full network coverage is ensured by NES over LMST neighbor set. Regardless of MAC layer problems, the way of reaching theses LMST neighbors has no impact on coverage. It means that these exist, in the same broadcasting task, nodes applying DLBOP and nodes applying OMDLBOP. The protocol we propose in next section is a solution where nodes locally decide independently between the two modes.

\subsection{Adaptive Directed LMST Broadcast Oriented Protocol (A-DLBOP)}

We are now in position to describe A-DLBOP algorithm which combines oneto-one and one-to-many communication models. The protocol A-DLBOP is a flooding protocol based on DLBOP and OM-DLBOP described above. Hence a node which receives the broadcasted messages starts a NES limited to LMST neighbors. At the end of the quiet period, the node has to choose between oneto-one or one-to-many communication models. For a given node $u$, the decision algorithm is the following one: 


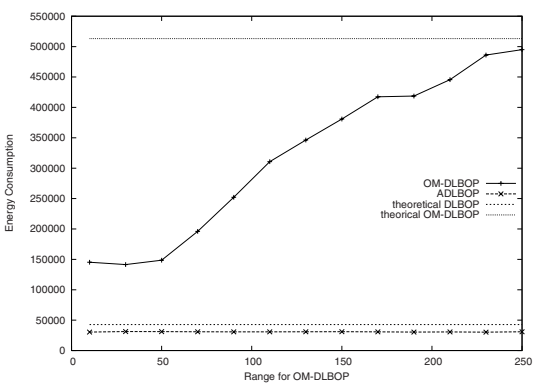

$\alpha=2, C_{1}=C_{2}=0$

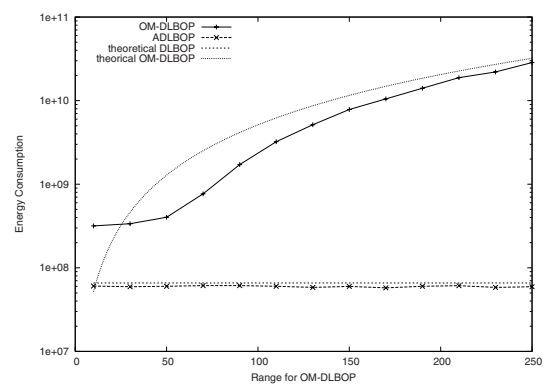

$\alpha>2, C_{1}=C_{2}=0$

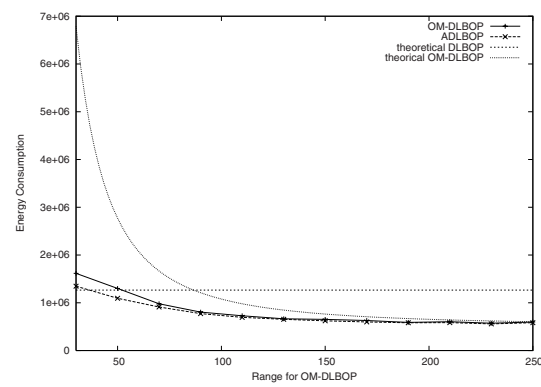

$\alpha=2, C_{1} \neq 0 \vee C_{2} \neq 0$

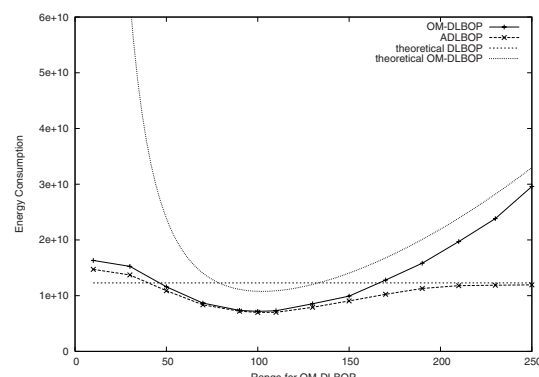

$\alpha>2, C_{1} \neq 0 \vee C_{2} \neq 0$

Fig. 5. Influence of $R_{o p t}$ on energy consumption.

- Let $A$ be the non-covered neighbors set and $B \subseteq A$ the non-covered LMST neighbors set. We denote by $A^{\prime}$ the set of nodes belonging to $A$ and closer than $R_{\text {opt }}(4 \pi / 3)$. As previously, if $C_{1}=C_{2}=0$ then $R_{\text {opt }}(4 \pi / 3)=0$. The "goal" of the node $u$ is to cover the set $C=A^{\prime} \cup B$. If the set $C$ is empty, the retransmission is canceled.

- The communication model choice is made from a comparison of energy consumption needed to cover $C$ :

- One-to-one communication: while flooding over the subset $C$ with oneto-one communication model, each node retransmits the message to its non-covered LMST neighbors. On average, each node has only one noncovered LMST neighbor. Hence the energy consumption with one-to-one communication model using $\beta$-beams can be evaluated by:

$$
E_{1-t o-1}=|C| \times e\left(\beta, d_{l m s t}\right) .
$$

The node $u$ ignores the distance $d_{l m s t}$ which represents the average LMST edge length. It can simply estimate it with distance between itself and its LMST neighbors:

$$
d_{l m s t} \simeq \frac{1}{|B|} \sum_{v \in B} d(u, v) .
$$


- One-to-many communication: let $\theta$ be the angle needed to cover $C$ (if $\overline{\theta<\beta}$ we consider that $\theta=\beta$ ) and let $d$ be the distance between node $u$ and the furthest node of $C$. The energy consumption of a single beam which covers $C$ is:

$$
E_{1-t o-\operatorname{many}}=e(\theta, d)
$$

- If $E_{1-t o-1}<E_{1-t o-m a n y}$, the node $u$ decides to use one-to-one communication model and sends a $\beta$-beam to each non-covered LMST neighbor (nodes of $B)$.

- Otherwise, the node $u$ decides to use one-to-many communication model and sends a $\theta$-beam to cover nodes of $C$. If $d<R_{\text {opt }}(\theta)$, the beam range is increased in order to reach neighbors of $A$ closer than $R_{o p t}(\theta)$.

Hence, the protocol A-DLBOP is an adaptive broadcast protocol where decision is made locally by each node. We present in next section experimental results for our three protocols DLBOP, OM-DLBOP and A-DLBOP and we compare them to the centralized DBIP protocol.

\section{Experimental Results}

\subsection{Validation of Optimal Transmission Radius Existence}

The two protocols OM-DLBOP and A-DLBOP use an optimal transmission radius function $R_{o p t}$ which depends on energy consumption model (parameters $\alpha, C_{1}, C_{2}$ ). This function $R_{o p t}$ has been theoretically studied in Section 4 and results are summarized in Table 1. The goal of the first experiments is to valid these results by replacing the function $R_{\text {opt }}$ by a value we control.

We use randomly generated networks of 500 nodes (only connected graphs are considered). The other parameters are the size of the square grid $S=$ $1000 \times 1000 \mathrm{~m}$ (for a density around of 78 nodes by communication zone) and the minimal angle beam $\beta=\pi / 9$. We evaluate theses instances for varying radius target $R_{o p t}$ from $10 \mathrm{~m}$ to the maximal range $(250 \mathrm{~m})$. NES timeout is randomly generated and the simulator uses an ideal MAC layer (with absence of collisions).

We compare the OM-DLBOP protocol and the A-DLBOP protocol, because they are the only ones which are influenced by the value of $R_{o p t}$. We add to the graphs the values of $E_{D L B O P}$ and $E_{O M-D L B O P}$ for the given configuration, to compare and justify the validity of the theoretical models.

The Figure 5 shows the impact of $R_{\text {opt }}$ on energy consumption for different protocols. The graphs presents the EER (Expanded Energy Ratio: the results are normalized in function of the best energy economy, which is equal to 100) for each enery model. Concerning the case where $\alpha=2$ and $C_{1}=C_{2}=0$, although that theoretical study indicates that the energy consumption does not vary with transmission radius (Table 1), experimentally, the OM-DLBOP energy consumptions increases. This is probably due to extra retransmissions from NES. This fact validates the choice of $R_{o p t}=0$ in OM-DLBOP and A-DLBOP protocols for this energy model. 

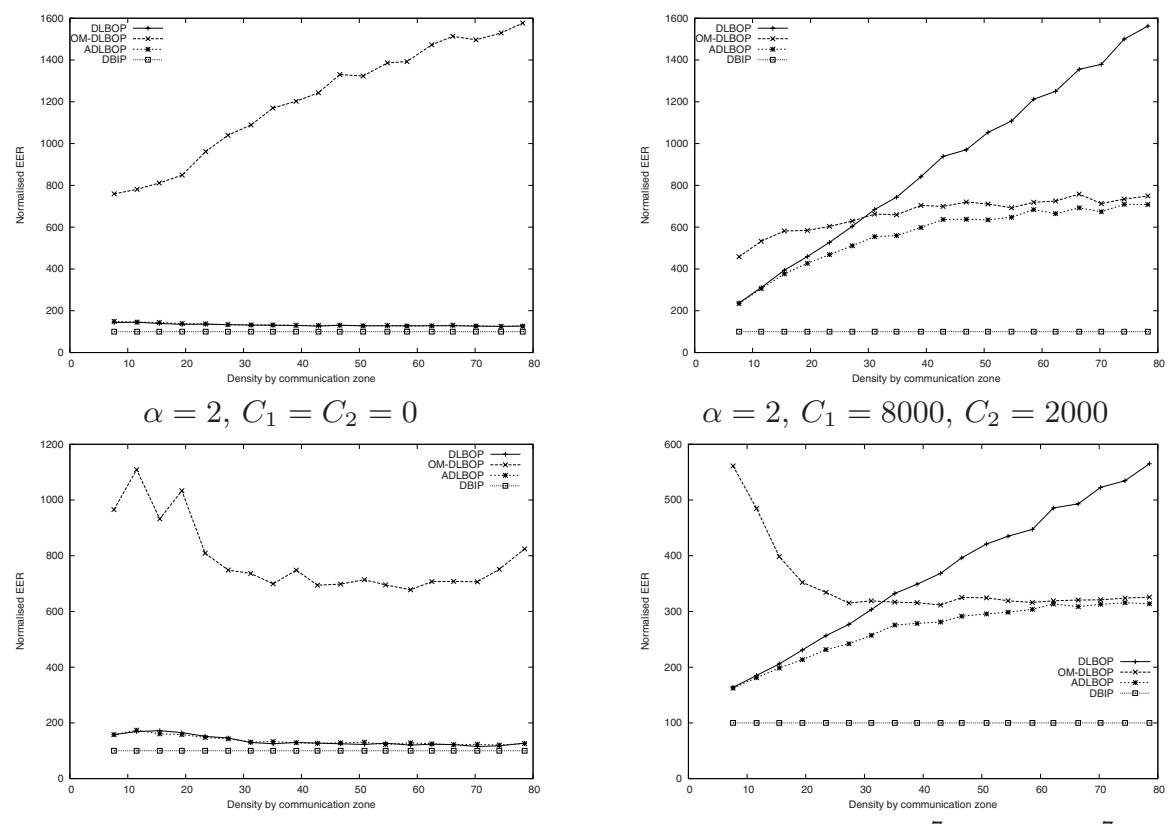

$\alpha=4, C_{1}=C_{2}=0$

$\alpha=4, C_{1}=8.10^{7}, C_{2}=2.10^{7}$

Fig. 6. Normalized Energy consumption for $S=1000 \times 1000$ and $\beta=\pi / 9$.

For the case $\alpha=2$ and $C_{1} \neq 0$ or $C_{2} \neq 0$, we have demonstrated that the best solution is to maximize $R_{\text {opt }}$. Our simulations show that the values of the OM-DLBOP protocol and A-DLBOP are very close, and offer results identical to the theoretical value of the OM-DLBOP mode.

For the energy model $\alpha>2$ and $C_{1}=C_{2}=0$ (presented with a logarithmic scale for the ordinate), we have again confirmed the theoretical model validity, as the OM-DLBOP protocol follows the values given by $E_{O M-D L B O P}$. Again, the best energy saving is given by the DLBOP method. More generally, if the constants are null, then the best solution is to minimize the radius of each sending, and so the DLBOP protocol is used. Even if the A-DLBOP model gives better results, it is not necessary the same case for other values of $\beta, n$ and $S$, as shown by the turning points between the theoretical models of DLBOP and OM-DLBOP.

For the last energy model, $\alpha>2$ and $C_{1} \neq 0$ or $C_{2} \neq 0$, the OM-DLBOP protocol reaches minimal value for $R_{o p t}=99$ (compared with the theoretical value of $\left.R_{o p t}=102.41\right)$. The A-DLBOP protocol succeed to adapt its behavior as function of $R_{\text {opt }}$ : it switches to the OM-DLBOP protocol when it becomes more interesting. Furthermore, once again, the experimental results follow the theoretical values. 


\subsection{Study of Adaptability: Energy Consumption Versus Network Density}

The experimental data for the four energy models are presented in Figure 6 . The following parameters are used: $S=1000 \times 1000 \mathrm{~m} ; \beta=\pi / 9$. The number of nodes varies from 50 to 500 according to the target density. The energy consumption is normalized as function of the best energy savings (which is equal to 100).

For the first energy model (with $\alpha=2$ and $C_{1}=C_{2}=0$ ), the OM-DLBOP protocol is inefficient, because it rapidly increases, compared to the others algorithms. This is the excepted behavior, as the energy model has zero constants. The localized DLBOP and A-DLBOP protocols give close results to globalized DBIP: from $26 \%$ to $33 \%$.

For the second energy model (with $\alpha=2, C_{1}=8000$ or $C_{2}=2000$ ), the radius target $R_{\text {opt }}$ is fixed to the maximal range $(250 \mathrm{~m})$. The choice is made following the theoretical analysis, which indicates that the best solution is to maximize the range. The graphs clearly show the advantage the interest of the A-DLBOP mode. When the density is lower than approximately 37 nodes by communication zone, the one-to-one mode is preferred. After this threshold, the protocol switches to a higher use of the one-to-many mode. This is confirmed by the theoretical result (eq. [5), despite the border effect (54 nodes for $\beta=\pi / 9$ ).

The third energy model (i.e. $\alpha=4$ and $C_{1}=C_{2}=0$ ) has similar behavior to the first energy model: the DLBOP mode is always better. More generally, with null constants, it is better to use DLBOP solution. In this case, the OM-DLBOP mode is inefficient.

For the last energy model $\left(\alpha=4, C_{1}=8.10^{7}\right.$ and $\left.C_{2}=2.10^{7}\right)$, the OMDLBOP algorithm is too energy-consuming for low densities. When the density increases, between 25 and 30 nodes by communication zone, the OM-DLBOP algorithm becomes better than DLBOP. The interesting fact is the A-DLBOP protocol takes advantages of the two communication models, by using them at the same time. Hence, A-DLBOP has better energy-saving than DLBOP and OM-DLBOP. The figure confirms the inequality ([6), with an experimental threshold value of 30 for a theoretical value of 35 . The other inequality (6) is also correct in respect of the figure.

\section{Conclusion}

We have proposed in this paper a new localized protocol called A-DLBOP, that combines one-to-one and one-to-many communication models. The first suffers from high overhead with high density, compared to DBIP. The second communication model corrects this problem by using variable angle to cover large number of nodes The protocol A-DLBOP uses either the first or the second model as function of a local evaluation of the energy consumption. We proposed a theoretical evaluation of the performance of the algorithm. These results are confirmed by the experimental data. 


\section{References}

1. Wieselthier, J., Nguyen, G., Ephremides, A.: On the construction of energy-efficient broadcast and multicast trees in wireless networks. In: Proc. IEEE INFOCOM 2000, Tel Aviv, Israel (2000) 585-594

2. Spyropoulos, A., Raghavendra, C.: Energy efficient communications in ad hoc networks using directional antennas. In: Proc. IEEE INFOCOM 2002, New-York, USA (2002)

3. Wieselthier, J., Nguyen, G., Ephremides, A.: Energy-limited wireless networking with directional antennas: the case of session-based multicasting. In: Proc. IEEE INFOCOM 2002, New-York, USA (2002)

4. Kirousis, L., Kranakis, E., Krizanc, D., Pelc, A.: Power consumption in packet radio networks. In R.Reischuk, M.Morvan, eds.: Proc. 14th Symposium on Theoretical Computer Science (STACS'97). Volume 1200 of Lecture Notes in Computer Science., Hansestadt Lübeck, Germany, Springer-Verlag, Berlin (1997) 363-374

5. Cartigny, J., Simplot, D., Stojmenović, I.: Localized minimum-energy broadcasting in ad-hoc networks. In: Proc. IEEE INFOCOM 2003, San Fransisco, USA (2003)

6. Cartigny, J., Simplot, D., Stojmenović, I.: Localized energy efficient broadcast for wireless networks with directional antennas. In: Proc. IFIP Mediterranean Ad Hoc Networking Workshop (MED-HOC-NET'2002), Sardegna, Italy (2002)

7. Toussaint, G.: The relative neighborhood graph of finite planar set. Pattern Recognition 12 (1980) 261-268

8. Li, N., Hou, J.: BLMST: A scalable, power-efficient broadcast algorithm for wireless sensor networks. Submitted (2002)

9. Cartigny, J., Ingelrest, F., Simplot-Ryl, D., Stojmenović, I.: Localized LMST and RNG based minimum-energy broadcast protocols in ad hoc networks. Ad Hoc Networks (2004) to appear.

10. Li, N., Hou, J., Sha, L.: Design and analysis of an MST-based topology control algorithm. In: Proc. IEEE INFOCOM 2003, San Francisco, USA (2003)

11. Rodoplu, V., Meng, T.: Minimum energy mobile wireless networks. In: IEEE J. Selected Area in Comm. Volume 17. (1999) 1333-1344

12. Peng, W., Lu, X.: On the reduction of broadcast redundancy in mobile ad hoc networks. In: Proc. Annual Workshop on Mobile and Ad Hoc Networking and Computing (MobiHOC 2000), Boston, Massachusetts, USA (2000) 129-130

13. Stojmenović, I., Seddigh, M., Zunic, J.: Dominating sets and neighbor elimination based broadcasting algorithms in wireless networks. IEEE Transactions on Parallel and Distributed Systems 13 (2002) 14-25

14. Ingelrest, F., Simplot-Ryl, D., Stojmenovic, I.: Target radius over LMST for energyefficient broadcast protocol in ad hoc networks. In: IEEE International Conference on Communications (ICC'2004), Paris, France (2004) 